\title{
Late Clinical Presentation of Tethered Cord Syndrome
}

\author{
Aziz Ahizoune, $M D^{1^{*}}$ and Hatim Belfquih, $M D^{2}$ \\ ${ }^{1}$ Specialist of Neurology, Department of Neurology, Avicenne Military Hospital of Marrakech, Morocco \\ ${ }^{2}$ Assistant Professor of Neurosurgery, Department of Neurosurgery, Avicenne Military Hospital of Marrakech, \\ Mohammed V University in Rabat, Morocco
}
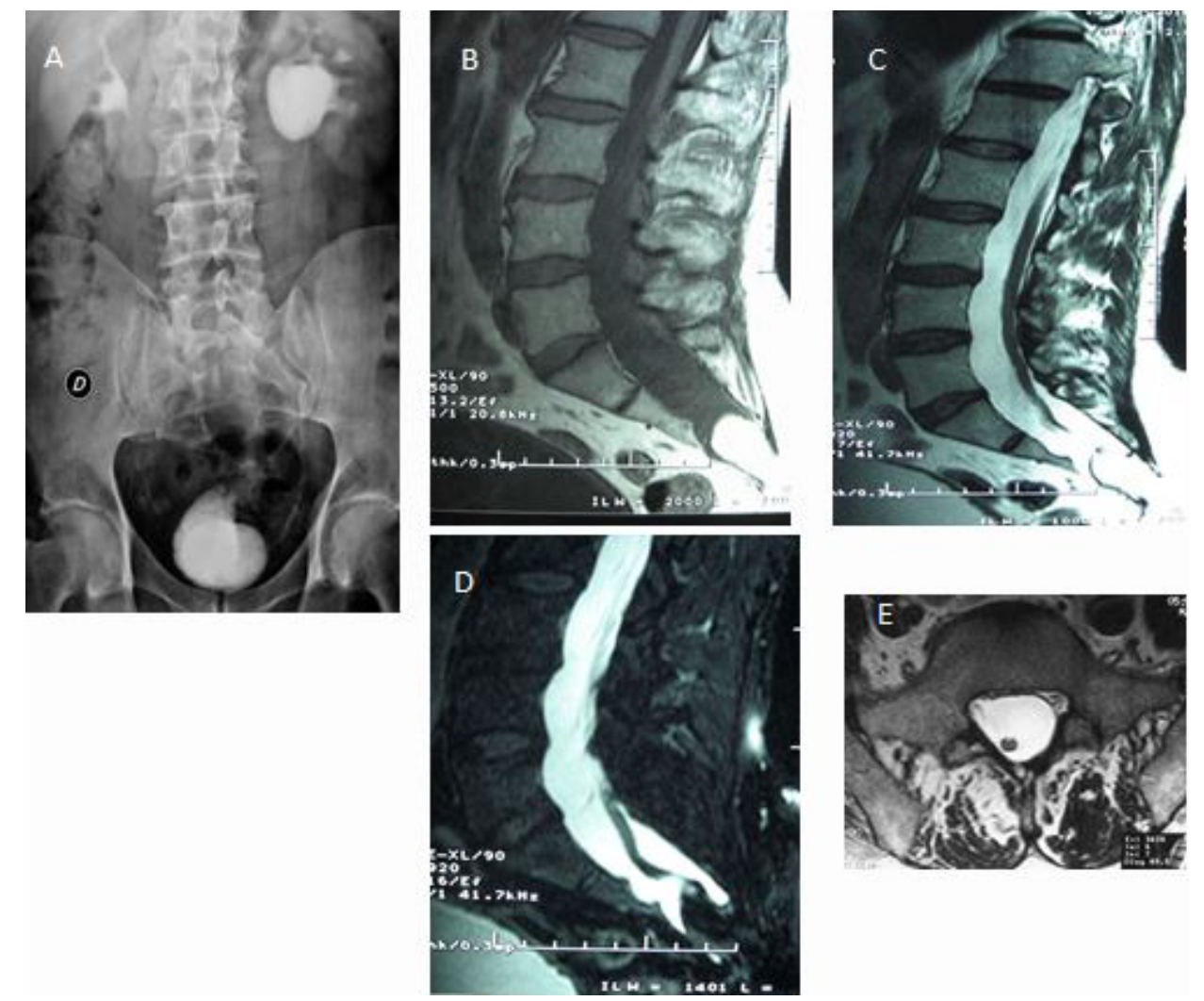

Figure 1: Tethered Cord Syndrome with sacral lipoma. (A) Intravenous Urography showing left pyelocalyceal dilatation associated to sacral spina bifida; B, C, D) Sagital MRI demonstrating tethered spinal cord attached to sacral lipoma which is hyperintense at T2, T1 sequences and in hypointense at FAT SAT T2 sequence; E) Axial T2 sequence at sacral lipoma level that is showing hyper-intensity.

Fifty six-years-old man with history of asthma and recurrent urinary tract infections, was presenting infrequent urine leakage for five years. The clinical examination was unremarkable. The urodynamic assessment revealed an overactive bladder with limited closing pressure. Intravenous Urography was performed and did not show any obstructive abnormalities, but we found out an incidental spina bifida of sacral vertebrae (Figure 1A). Therefore, a spinal MRI was performed and showed a tethered cord syndrome with intra-dural lipoma of the terminal filum (Figure 1B, Figure $1 \mathrm{C}$, Figure $1 \mathrm{D}$ and Figure $1 \mathrm{E})$. After oxybutinechlorhydrate as medical treatment, our patient showed marked symptom relief without recurrence for 12 months at follow up.
TCS comprises symptoms that results of fixation and excess strain on the spinal cord caused by stretching. Common symptoms include pain, motor deficits, sensory deficits, bladder dysfunction, and bowel dysfunction [1]. This affection

*Corresponding author: Aziz Ahizoune, MD, Specialist of Neurology, Department of Neurology, Avicenne Military Hospital, 40000, Marrakech, Morocco, Tel: +(212)-6-62-27-40-02

Accepted: June 15, 2019

Published online: June 17, 2019

Citation: Ahizoune A, Belfquih H (2020) Late Clinical Presentation of Tethered Cord Syndrome. J Neurosurg Res Rev 3(1):33-34 
is very uncommon in adults and detethering is generally recommended if patients have deteriorating symptoms. Asymptomatic patients with an incidentally found tethered cord should be closely monitored for onset of symptoms as our case [2].

\section{References}

1. Shokei Yamada, Daniel J Won (2007) What is the true tethered cord syndrome? Childs Nerv Syst 23: 371-375.

2. Kyle P O'Connor, Adam D Smitherman, Camille K Milton, et al. (2020) Surgical Treatment of Tethered Cord Syndrome in Adults: A Systematic Review and Meta-Analysis. World Neurosurgery 137: e221-e241. 